\title{
Integrated Guidance and Control of Multiple Interceptor Missiles Based on Improved Distributed Cooperative Control Strategy
}

Xiang Liu*1, Xiaogeng Liang ${ }^{1}$

Liu X (i) https: / / orcid.org/0000-0003-4456-2091 Liang X (iD https://orcid.org/0000-0002-7153-6668

\section{How to cite}

Liu X; Liang X (2019) Integrated Guidance and Control of Multiple Interceptor Missiles Based on Improved Distributed Cooperative Control Strategy. J Aerosp Technol Manag, 11: e2119. https:// doi.org/10.5028/jatm.v11.1003

\begin{abstract}
In this study, an improved cooperative integrated guidance and control (IGC) design method is proposed based on distributed networks to address the guidance and control problem of multiple interceptor missiles. An IGC model for a leading interceptor is constructed based on the relative kinematic relations between missiles and a target and the kinematic equations of the missiles in a pitch channel. The unknown disturbances of the model are estimated using a finite-time disturbance observer (FTDO). Then, the control algorithm for the leading interceptor is designed according to the disturbance estimation and nonsingular fast dynamic surface sliding mode control (SMC). To enhance the rate of convergence of the cooperative control commands for the interceptors, an improved cooperative control strategy is proposed based on the leader-follower distributed network. Consequently, the two velocity components of the interceptor in the pitch channel can be obtained, which are subsequently converted to the total velocity and flight path angle commands of the interceptor using kinematic relations. The control algorithm for the following interceptor is similarly designed using an FTDO and dynamic surface SMC. The effectiveness of the improved distributed cooperative control strategy for multiple interceptors is validated through simulations.
\end{abstract}

KEYWORDS: Integrated guidance and control, Finite-time disturbance observer (FTDO), Nonsingular fast dynamic surface sliding mode, Distributed network, Improved cooperative control strategy

\section{INTRODUCTION}

With the advancement of anti-missile technology, cooperative multi-missile attack and defense is attracting increasing attention owing to its unique strengths. As a result, the development of cooperative multi-missile guidance and control technology, which is a key element for ensuring the attack and defense performance of a weapon system, has gained momentum. Through coordination between missiles, cooperative engagement integrates multiple interceptor missiles as a united combat group that is informationsharing, function-complementary, and tactics-cooperative. Using the group advantage, a multi-missile system can execute a multilayer all-around attack on an enemy's defense system or a target with overall-promoted penetration capabilities and carries out tasks that are difficult for a single interceptor missile to perform. Therefore, it is of practical significance to study the cooperative guidance and control of multiple interceptors (Zhao and Yang 2017; Daughtery and Qu 2014).

With respect to the cooperative guidance and control of multi-interceptors, Jeon et al. (2010) and Lee et al. (2007) propose a guidance law with controllable attack time and angle-of-attack constraint and apply it to the salvo attack of anti-ship missiles.

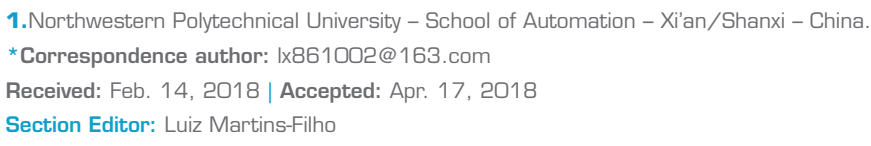


Based on this idea, researchers subsequently introduced other guidance and control methods, including sliding mode control (SMC) (Hail and Balakrishnan 2012; Cho et al. 2016), optimal control (Nikusokhan and Nobahari 2016), differential game (Kang and Kim 2011), and dynamic surface control (Wang et al. 2015). These methods rely on specifying attack time before launching to achieve coordination. As no information exchange occurs between missiles during flight, these methods apparently have temporal limitations. With progress in the consensus of multi-agent systems, researchers have begun to use the consensus theory to study the cooperative guidance and control of multi-interceptors. Using the coordination strategy under the cooperative guidance framework, Kumar and Ghose (2014) adjust missile trajectories so that the coordination variable of each missile can approach the expected coordination variable for realizing cooperative guidance. Zhao et al. (2014) apply leader-follower formation control to the cooperative guidance of multi-interceptors by employing an analogous leader-follower cooperative guidance framework. Sun et al. (2014) and Zhao et al. (2016) explore the guidance and control law of the leader-follower topology considering time delay and topology switch. By constructing an integrated cost function for multiple missiles, Shaferman and Shima (2015) design a cooperative guidance law for multiple missiles for intercepting a maneuvering target. However, the application of this function is faced with multiple constraints, as each missile requires the global information of all participating partners. Balhance et al. (2017) study the cooperative guidance law using the optimal control theory and improves communication between multiple missiles. These consensus-based cooperative guidance and control approaches typically employ the regular form of the cooperation strategy and fail to consider the convergence rate of interceptors to cooperative control commands.

Conventionally, in the design of the guidance and control system of interceptors, a control loop is set as a fast loop whereas a guidance loop is set as a slow loop. The basis of these methods is to design two subsystems independently, without considering the coupling between the two loops. On the contrary, integrated guidance and control (IGC) design depends on the control force generated from the engagement information between an interceptor and a target and the kinematic information of the interceptor per se to drive the interceptor to hit the target. This can ensure the stability of interceptor flight attitude and improve guidance accuracy (Menon and Ohlmeyer 2001; Shtessel et al. 2009). In recent years, researchers have integrated IGC design with various control theories, such as SMC (Shtessel et al. 2009; Lee et al. 2016), back-stepping control (Seyedipour et al. 2017; Ran et al. 2014), optimal control (Vaddi et al. 2009), and predictive control (Shamaghdari et al. 2015), for creating a suite of aircraft IGC design methods with diverse features. Even though it is well known that the advantages of IGC methods can be utilized to enhance the stability of multi-interceptor systems, research in this area is still inadequate at the moment.

Therefore, by adopting the above-discussed IGC method, an improved distributed cooperative IGC algorithm for multiple interceptor missiles featuring the leader-follower topology is proposed in this study based on the distributed network cooperative control strategy and SMC theory, with the aim of improving the rate of convergence of inceptor missiles to cooperative control commands.

\section{IGC MODEL FOR THE LEADING INTERCEPTOR}

The engagement geometry of the leading interceptor and target in the pitch channel are shown in Fig. 1.

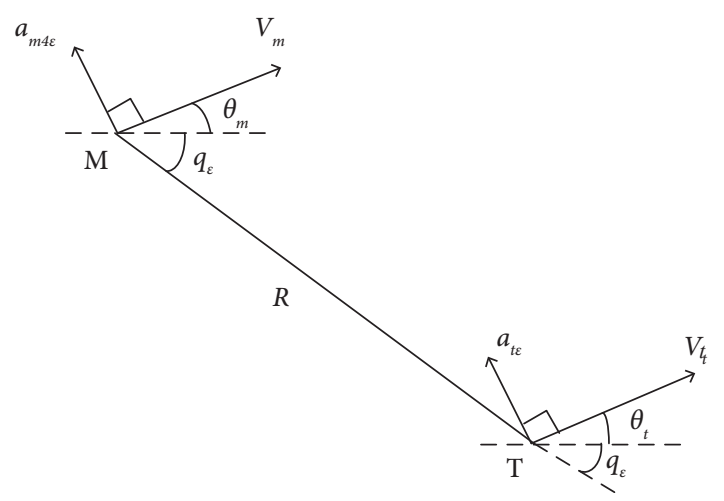

Figure 1. Engagement geometry of the leading interceptor and target. 
In Fig. 1, $M$ and $T$ denote the interceptor missile and target, respectively; $a_{m 4 \varepsilon}$ and $a_{t \varepsilon}$ are the accelerations of the missile and target, respectively; $V_{m}$ and $V_{t}$ represent missile velocity and target velocity, respectively; $\theta_{m}$ and $\theta_{t}$ are the flight path angles of the missile and target, respectively; $q_{\varepsilon}$ is the missile/target LOS angle; $R$ is the distance between the missile and target. The relative kinematic model of the interceptor in the longitudinal plane can be obtained as (Eq. 1):

$$
\left\{\begin{array}{l}
\dot{r}=V_{t} \cos \left(q_{\varepsilon}-\theta_{t}\right)-V_{m} \cos \left(q_{\varepsilon}-\theta_{m}\right) \\
r \dot{q}_{\varepsilon}=V_{m} \sin \left(q_{\varepsilon}-\theta_{m}\right)-V_{t} \sin \left(q_{\varepsilon}-\theta_{t}\right)
\end{array}\right.
$$

The kinematic model of the leading interceptor in the pitch channel is expressed as (Eq. 2):

$$
\left\{\begin{array}{l}
\dot{\alpha}=\omega_{z}-\frac{q S C_{y}^{\alpha} \alpha \cos \alpha}{m V_{m}}+d_{\alpha} \\
\omega_{z}=\frac{q S \bar{L} m_{z}^{\alpha} \alpha}{J_{z}}+\frac{q S \bar{L} m_{z}^{\omega_{z}} \omega_{Z}}{J_{z}}+\frac{M_{z}}{J_{z}}+d_{\omega_{z}} \\
a_{m 3 \varepsilon}=\frac{q S C_{y}^{\alpha} \alpha}{m}
\end{array}\right.
$$

where $S$ is the reference area of the missile; $m$ the missile mass; $\alpha$ is the angle of attack (AOA); $\omega_{z}$ is the pitch angular velocity; $q$ is the dynamic pressure; $d_{\alpha}$ and $d_{\omega_{z}}$ are the perturbation and uncertain disturbance in each part of the system, respectively; $J_{z}$ is the rotational inertia of the missile; $C_{y}^{a}, m_{z}^{a}$, and $m_{z}^{\omega_{z}}$ denote the aerodynamic force and moment coefficients; and $M_{z}$ is the control moment of the missile in the pitch channel.

Based on Eqs. 1 and 2, if we define $x_{1}=q_{\varepsilon}, x_{2}=\dot{q}_{\varepsilon}, x_{3}=\alpha$, and $x_{4}=\omega_{z}$, the nonlinear IGC model of the leading interceptor in the pitch channel can be written as (Eq. 3):

$$
\left\{\begin{array}{l}
\dot{x}_{1}=x_{2} \\
\dot{x}_{2}=a_{22} x_{2}+a_{23} x_{3}+\frac{a_{t \varepsilon}}{r} \\
\dot{x}_{3}=a_{33} x_{3}+x_{4}+d_{\alpha} \\
\dot{x}_{4}=a_{43} x_{3}+a_{44} x_{4}+b_{4} u+d_{\omega_{z}} \\
y_{2}=x_{2}
\end{array}\right.
$$

where $\quad a_{22}=-\frac{2 \dot{r}}{r}, \quad a_{23}=-\frac{q S C_{y}^{\alpha}}{m r}, \quad a_{33}=-\frac{q S C_{y}^{\alpha}}{m V_{m}}, \quad a_{43}=\frac{q S \bar{L} m_{z}^{\alpha}}{J_{z}}, \quad a_{44}=\frac{q S \bar{L} m_{z}^{\omega_{z}}}{J_{z}}, \quad b_{4}=\frac{1}{J_{z}}$, and $u=M_{z}$.

Assumption 1: The LOS angle of the interceptor varies insignificantly during the terminal phase of guidance. In addition, the angle between the SOL direction and velocity direction is small; hence, $a_{m 3 \varepsilon} \approx a_{m 4 \varepsilon}$.

Assumption 2: The unknown disturbances in the IGC system, $d_{\alpha}$ and $d_{\mathrm{w}_{z}}$, are continuously differentiable and have a bounded first derivative, i.e., $d_{i}<L$, where $L$ is a positive constant.

\section{FTDO DESIGN}

An FTDO estimates the nonlinear uncertain terms in the system model and feeds their estimated values into the control system for compensation. To eliminate the effects of the unknown disturbances involved in model (Eq. 3 ), viz., $a_{t \varepsilon}, d_{\alpha}$, and $d_{\omega_{z}}$, on the control system of the leading interceptor, an FTDO is designed to estimate these terms.

Defining $v_{r}=\dot{r}$ and ve $=r \dot{q}_{\varepsilon}$, from Eq. 1 , we have (Eq. 4$)$ 


$$
\dot{v}_{\varepsilon}=\dot{r} \dot{q}_{\varepsilon}+r \ddot{q}_{\varepsilon}=\dot{r} \dot{q}_{\varepsilon}-2 \dot{r}_{\varepsilon}+a_{t \varepsilon}-a_{m 4 \varepsilon}=-\frac{v_{r} v_{\varepsilon}}{r}+a_{t \varepsilon}-a_{m 4 \varepsilon}
$$

The longitudinal acceleration of the target, $a_{t \varepsilon}$, can be estimated using the following FTDO (Eq. 5):

$$
\left\{\begin{array}{l}
\dot{z}_{20}=v_{20}+g_{2}, \dot{z}_{21}=v_{21}, \dot{z}_{22}=v_{22} \\
v_{20}=-\lambda_{20} L_{2}^{1 / 3}\left|z_{20}-v_{\varepsilon}\right|^{2 / 3} \operatorname{sgn}\left(z_{20}-v_{\varepsilon}\right)-\sigma_{20}\left(z_{20}-v_{\varepsilon}\right)+z_{21} \\
v_{21}=-\lambda_{21} L_{2}^{1 / 2}\left|z_{21}-v_{20}\right|^{1 / 2} \operatorname{sgn}\left(z_{21}-v_{20}\right)-\sigma_{21}\left(z_{21}-v_{20}\right)+z_{22} \\
v_{22}=-\lambda_{22} L_{2}\left|z_{22}-v_{21}\right|^{q_{2} / p_{2}} \operatorname{sgn}\left(z_{22}-v_{21}\right)-\sigma_{22}\left(z_{22}-v_{21}\right) \\
z_{20}=\tilde{v}_{\varepsilon}, z_{21}=\tilde{a}_{t \varepsilon}, z_{22}=\tilde{a}_{t \varepsilon}
\end{array}\right.
$$

where $g_{2}=-\left(v_{r} v_{\varepsilon} / r\right)-\left(q S C_{y}^{a} / m\right) a ; \tilde{v}_{\varepsilon}$ and $\tilde{a}_{t \varepsilon}$ are the disturbance estimates of $v_{\varepsilon}$ and $a_{t \varepsilon}$, respectively; $\lambda_{20}, \lambda_{21}, \lambda_{22}, \sigma_{20}, \sigma_{21}$, and $\sigma_{22}$ are the design parameters of the FTDO; $q_{2}$ and $p_{2}$ are terminal parameters with $0<p_{2}<q_{2}$.

According Shtessel et al. (2007), the FTDO error system is stable during finite time; thus, we define the estimation error of the target acceleration as $e_{21}=z_{21}-a_{t \varepsilon}$.

Similarly, the disturbances of the leading interceptor in the AOA loop and pitch angular velocity loop, $d_{\alpha}$ and $d_{\omega_{z}}$, can be estimated by (Eqs. 6 and 7):

$$
\begin{aligned}
& \left\{\begin{array}{l}
\dot{z}_{30}=v_{30}+g_{3}, \dot{z}_{31}=v_{31}, \dot{z}_{32}=v_{32} \\
v_{30}=-\lambda_{30} L_{3}^{1 / 3}\left|z_{30}-x_{3}\right|^{2 / 3} \operatorname{sgn}\left(z_{30}-x_{3}\right)-\sigma_{30}\left(z_{30}-x_{3}\right)+z_{31} \\
v_{31}=-\lambda_{31} L_{3}^{1 / 2}\left|z_{31}-v_{30}\right|^{1 / 2} \operatorname{sgn}\left(z_{31}-v_{30}\right)-\sigma_{31}\left(z_{31}-v_{30}\right)+z_{32} \\
v_{32}=-\lambda_{32} L_{3}\left|z_{32}-v_{31}\right|^{q_{3} / p_{3}} \operatorname{sgn}\left(z_{32}-v_{31}\right)-\sigma_{32}\left(z_{32}-v_{31}\right) \\
z_{30}=\tilde{x}_{3}, z_{31}=\tilde{d}_{\alpha}, z_{32}=\tilde{\dot{d}}_{\alpha}
\end{array}\right. \\
& \left\{\begin{array}{l}
\dot{z}_{40}=v_{40}+g_{4}, \dot{z}_{41}=v_{41}, \dot{z}_{42}=v_{42} \\
v_{40}=-\lambda_{40} L_{4}^{1 / 3}\left|z_{40}-x_{4}\right|^{2 / 3} \operatorname{sgn}\left(z_{40}-x_{4}\right)-\sigma_{40}\left(z_{40}-x_{4}\right)+z_{41} \\
v_{41}=-\lambda_{41} L_{4}^{1 / 2}\left|z_{41}-v_{40}\right|^{1 / 2} \operatorname{sgn}\left(z_{41}-v_{40}\right)-\sigma_{41}\left(z_{41}-v_{40}\right)+z_{42} \\
v_{42}=-\lambda_{42} L_{4}\left|z_{42}-v_{41}\right|^{q_{4} / p_{4}} \operatorname{sgn}\left(z_{42}-v_{41}\right)-\sigma_{42}\left(z_{42}-v_{41}\right) \\
z_{40}=\tilde{x}_{4}, z_{41}=\tilde{d}_{\omega_{z}}, z_{32}=\tilde{\dot{d}}_{\omega_{z}}
\end{array}\right.
\end{aligned}
$$

where $g_{3}=a_{33} x_{3}+x_{4} ; g_{4}=a_{43} x_{3}+a_{44} x_{4}+b_{4} u ; \tilde{d}_{a}$ and $\tilde{d}_{\omega_{z}}$ are the estimated values of $d_{\alpha}$ and $d_{\omega_{z}}$ with estimation errors of $e_{31}=z_{31}-d_{\alpha}$ and $e_{41}=z_{41}-d_{\omega_{z}}$, respectively.

\section{DESIGN OF THE NONSINGULAR FAST DYNAMIC SMC CONTROLLER}

The interceptor IGC model is a mismatched uncertain system; we designed a nonsingular fast dynamic surface SMC model as the control algorithm for the leading interceptor, based on the IGC model (Eq. 3) and FTDO estimations (Eqs. 5 to 7 ).

- According to dynamic surface SMC design, the second subsystem in Eq. 3 is considered. $x_{2 d}$ is defined as the system tracking command signal.

The first dynamic error surface is defined as (Eq. 8):

$$
s_{2}=-a_{23}^{-1}\left(x_{2}-x_{2 d}\right)
$$


The derivative of $s_{2}$ is determined to obtain the error dynamic equation (Eq. 9):

$$
\dot{s}_{2}=a_{23}^{-2} \dot{a}_{23}\left(x_{2}-x_{2 d}\right)-a_{23}^{-1}\left(a_{22} x_{2}+\frac{a_{t \varepsilon}}{r}-\dot{x}_{2 d}\right)-x_{3}
$$

The FTDO-estimated $\tilde{a}_{t \varepsilon}$ from Eq. 5 is substituted into Eq. 9 to acquire the virtual control input of the first dynamic surface (Eq. 10):

$$
x_{3}^{*}=a_{23}^{-2} \dot{a}_{23}\left(x_{2}-x_{2 d}\right)+k_{2} s_{2}-a_{23}^{-1}\left(a_{22} x_{2}+\frac{\tilde{a}_{t \varepsilon}}{r}-\dot{x}_{2 d}\right)
$$

where $k_{2}>0$. To prevent the increase in computational complexity owing to the 'explosion of terms' when finding the derivative of the virtual control input, $x_{3}^{*}$ is fed through a first-order low-pass filter to obtain the filtered virtual control input (Eq. 11):

$$
\tau_{3} \dot{\bar{x}}_{3}^{*}+\bar{x}_{3}^{*}=x_{3}^{*}, \bar{x}_{3}^{*}(0)=x_{3}^{*}(0)
$$

where $\tau_{3}$ is the time constant of the filter. Hence, the derivative of the virtual control input after error surface filtering is (Eq. 12):

$$
\dot{\bar{x}}_{3}^{*}=-\tau_{3}^{-1}\left(\bar{x}_{3}^{*}-x_{3}^{*}\right)
$$

- The second dynamic error surface is defined as (Eq. 13):

$$
s_{3}=x_{3}-\bar{x}_{3}^{*}
$$

The derivative of $s_{3}$ is determined to obtain the error dynamic equation (Eq. 14):

$$
\dot{s}_{3}=\dot{x}_{3}-\dot{\bar{x}}_{3}^{*}=a_{33} x_{3}+x_{4}+d_{\alpha}-\dot{\bar{x}}_{3}^{*}
$$

Based on the design approach of the first dynamic surface, the FTDO-estimated $\tilde{d}_{a}$ from Eq. 6 is substituted into Eq. 14 to acquire the virtual control input for the second dynamic surface (Eq. 15):

$$
x_{4}^{*}=-a_{33} x_{3}-\tilde{d}_{\alpha}+\dot{\bar{x}}_{3}^{*}-k_{3} s_{3}
$$

where $k_{3}>0 . x_{4}^{*}$ is fed through a low-pass filter to obtain (Eq. 16):

$$
\tau_{4} \dot{\bar{x}}_{4}^{*}+\bar{x}_{4}^{*}=x_{4}^{*}, \bar{x}_{4}^{*}(0)=x_{4}^{*}(0)
$$

where $\tau_{4}$ is the time constant of the filter. Hence, the derivative of the virtual control input after error surface filtering is (Eq. 17):

$$
\dot{\bar{x}}_{4}^{*}=-\tau_{4}^{-1}\left(\bar{x}_{4}^{*}-x_{4}^{*}\right)
$$

- The third dynamic error surface is defined as (Eq. 18):

$$
s_{4}=x_{4}-\bar{x}_{4}^{*}
$$

The derivative of $s_{4}$ is determined to obtain the error dynamic equation (Eq. 19):

$$
\dot{s}_{4}=\dot{x}_{4}-\dot{\bar{x}}_{4}^{*}=a_{43} x_{3}+a_{44} x_{4}+b_{4} u+d_{\omega_{z}}-\dot{\bar{x}}_{4}^{*}
$$


To prevent singularity in the leading interceptor system and to converge to the equilibrium position within limited time, we design a nonsingular fast sliding mode reaching law (Eq. 20):

$$
\dot{s}=-\left|x_{a}\right|^{p / q-1}\left[k_{a} s+k_{b}|s|^{\partial} \operatorname{sgn}(s)\right]
$$

where $p, q \in N^{+}$and odd, $1<p / q<2, k_{a}>0, k_{b}>0$, and $0<\partial<1$.

Based on Eqs. 19 and 20 and the FTDO-estimated $\tilde{d}_{\omega_{z}}$ from Eq. 7, the improved nonsingular fast dynamic surface SMC law of the leading interceptor is (Eq. 21):

$$
u=b_{4}^{-1}\left[-a_{43} x_{3}-a_{44} x_{4}-\tilde{d}_{\omega_{z}}+\dot{\bar{x}}_{4}^{*}-k_{4}\left|x_{4}\right|^{p / q-1} s_{4}-k_{5}\left|x_{4}\right|^{p / q-1}\left|s_{4}\right|^{\partial} \operatorname{sgn}\left(s_{4}\right)\right]
$$

where $k_{4}>0, k_{5}>0,1<p / q<2$, and $0<\partial<1$.

\section{STABILITY ANALYSIS}

It is assumed that the estimation errors of the FTDO system satisfy (Eq. 22):

$$
\left|e_{21}\right|<N_{2},\left|e_{31}\right|<N_{3},\left|e_{41}\right|<N_{4}
$$

where $N_{2}, N_{3}$, and $N_{4}$ are positive constants.

Filtering errors are defined as follows (Eq. 23):

$$
y_{3}=\bar{x}_{3}^{*}-x_{3}^{*}, y_{4}=\bar{x}_{4}^{*}-x_{4}^{*}
$$

The derivatives of $y_{3}$ and $y_{4}$ are determined to obtain the dynamic errors of filtering (Eq. 24):

$$
\dot{y}_{3}=-\tau_{3}^{-1} y_{3}-\dot{x}_{3}^{*}, \dot{y}_{4}=-\tau_{4}^{-1} y_{4}-\dot{x}_{4}^{*}
$$

Based on Eqs. 8 to 18 and 23, we have:

$$
\left\{\begin{array}{l}
x_{2}=-a_{23} s_{2}+x_{2} d \\
x_{3}=s_{3}+\bar{x}_{3}^{*}=s_{3}+y_{3}+x_{3}^{*} \\
x_{4}=s_{4}+\bar{x}_{4}^{*}=s_{4}+y_{4}+x_{4}^{*}
\end{array}\right.
$$

Based on Eqs. 3, 8 to 18, and 23 to 25 , we have (Eq. 26 to 28 ):

$$
\dot{s}_{2}=a_{23} \dot{a}_{23}\left(x_{2}-x_{2 d}\right)-a_{23}^{-1}\left(a_{22} x_{2}+\frac{a_{t \varepsilon}}{r}-\dot{x}_{2 d}\right)-s_{3}-y_{3}-x_{3}^{*}=-s_{3}-y_{3}-k_{2} s_{2}+\tilde{e}_{21}
$$

where $\tilde{e}_{21}=\frac{m}{q S C_{y}^{\alpha}}\left(\tilde{a}_{t \varepsilon}-a_{t \varepsilon}\right)$.

It is assumed that $\left|\tilde{e}_{21}\right|<\tilde{N}_{2}$, where $\tilde{N}_{2}$ is a positive constant.

$$
\begin{aligned}
& \dot{s}_{3}=a_{33} x_{3}+s_{4}+y_{4}+x_{4}^{*}+d_{\alpha}-\dot{x}_{3}^{*}=s_{4}+y_{4}-k_{3} s_{3}-e_{31} \\
& \dot{s}_{4}=a_{43} x_{3}+a_{44} x_{4}+b_{4} u+d_{\omega_{z}}-x_{4}^{*}=-k_{4}\left|x_{4}\right|^{p / q-1} s_{4}-k_{5}\left|x_{4}\right|^{p / q-1}\left|s_{4}\right|^{\partial} \operatorname{sgn}\left(s_{4}\right)-e_{41}
\end{aligned}
$$


As all variables and their derivatives in the system model are bounded, there exist continuous functions $\tilde{z}_{3}<0$ and $\tilde{z}_{4}<0$ such that variables $\dot{x}_{3}^{*}$ and $\dot{x}_{4}^{*}$ satisfy (Eq. 29):

$$
\begin{aligned}
& \left|\dot{x}_{3}^{*}\right| \leq \tilde{z}_{3}\left(s_{2}, s_{3}, y_{3}, y_{4}, e_{21}, e_{31}, k_{2}, k_{3}, x_{2 d}, \dot{x}_{2 d}, \ddot{x}_{2 d}\right) \\
& \left|\dot{x}_{4}^{*}\right| \leq \tilde{z}_{4}\left(s_{2}, s_{3}, s_{4}, y_{3}, y_{4}, e_{21}, e_{31}, k_{2}, k_{3}, x_{2 d}, \dot{x}_{2 d}, \ddot{x}_{2 d}\right)
\end{aligned}
$$

Given constants $\chi$ and $R^{*}$, where $\chi>0$ and $R^{*}>0$, the following compact sets are defined: $B_{2}=\left[x_{2 d}, \dot{x}_{2 d}, \ddot{x}{ }_{2 d}\right]^{T}$, $x_{2 d}+\dot{x}_{2 d}+\ddot{x}_{2 d} \leq \chi$ and $B_{2}=\left[s_{2}, s_{3}, s_{4}, y_{3}, y_{4}, e_{21}, e_{31}\right]^{T}, s_{2}{ }^{2}, s^{2}, s^{2}{ }_{4}, y_{3}{ }_{3}, y_{4}^{2} \leq R^{*}$. Moreover, we know that $B_{1} \times B_{2}$ is a compact set. Let constants $M_{3}$ and $M_{4}$ be the maxima of $\tilde{z}_{3}$ and $\tilde{z}_{4}$ on $B_{1} \times B_{2}$, respectively, where $M_{3}>0$ and $M_{4}>0$. We have (Eq. 30):

$$
\left|\dot{x}_{3}^{*}\right| \leq M_{3},\left|\dot{x}_{4}^{*}\right| \leq M_{4}
$$

Based on Eqs. 25 to 28, the following can be obtained (Eqs. 31 to 35 ):

$$
\begin{gathered}
s_{2} \dot{s}_{2}=s_{2}\left(-s_{3}-y_{3}-k_{2} s_{2}+\tilde{e}_{21}\right) \leq\left(\frac{3}{2}-k_{2}\right) s_{2}^{2}+\frac{1}{2} s_{3}^{2}+\frac{1}{2} y_{3}^{2}+\frac{1}{2} \tilde{N}_{2}^{2} \\
s_{3} \dot{s}_{3}=s_{3}\left(s_{4}+y_{4}-k_{3} s_{3}-e_{31}\right) \leq\left(\frac{3}{2}-k_{3}\right) s_{3}^{2}+\frac{1}{2} s_{4}^{2}+\frac{1}{2} y_{4}^{2}+\frac{1}{2} N_{3}^{2} \\
s_{4} \dot{s}_{4}=s_{4}\left(-k_{4}\left|x_{4}\right|^{p / q-1} s_{4}-k_{5}\left|x_{4}\right|^{p / q-1}\left|s_{4}\right|^{\partial} \operatorname{sgn}\left(s_{4}\right)-e_{41}\right) \leq-k_{4}\left|x_{4}\right|^{p / q-1} s_{4}^{2}-k_{5}\left|x_{4}\right|^{p / q-1}\left|s_{4}\right|^{\partial+1}+\frac{1}{2} s_{4}^{2}+\frac{1}{2} e_{41}^{2} \leq 1 \\
\leq\left(\frac{1}{2}-k_{4}\left|x_{4}\right|^{p / q-1}+\frac{3}{2} k_{5}\left|x_{4}\right|^{p / q-1}\right) s_{4}^{2}+\frac{1}{2} N_{4}^{2}+\frac{1}{2} k_{5}\left|x_{4}\right|^{p / q-1} \\
y_{3} \dot{y}_{3}=y_{3}\left(-\tau_{3}^{-1} y_{3}-\dot{x}_{3}^{*}\right) \leq-\tau_{3}^{-1} y_{3}^{2}+\frac{1}{2}\left(y_{3}^{2}+M_{3}^{2}\right) \\
y_{4} \dot{y}_{4}=y_{4}\left(-\tau_{4}^{-1} y_{4}-\dot{x}_{4}^{*}\right) \leq-\tau_{4}^{-1} y_{4}^{2}+\frac{1}{2}\left(y_{4}^{2}+M_{4}^{2}\right)
\end{gathered}
$$

According to the nonlinear IGC model in Eq. 3, the Lyapunov function is considered as:

$$
V=\frac{1}{2}\left(s_{2}^{2}+s_{3}^{2}+s_{4}^{2}+y_{3}^{2}+y_{4}^{2}\right)
$$

The derivatives of both sides of Eq. (36) are determined to obtain (Eq. 37):

$$
\begin{aligned}
\dot{V}= & s_{2} \dot{s}_{2}+s_{3} \dot{s}_{3}+s_{4} \dot{s}_{4}+y_{3} \dot{y}_{3}+y_{4} \dot{y}_{4} \leq\left(\frac{3}{2}-k_{2}\right) s_{2}^{2}+\frac{1}{2} s_{3}^{2}+\frac{1}{2} y_{3}^{2}+\frac{1}{2} \tilde{N}_{2}^{2}+\left(\frac{3}{2}-k_{3}\right) s_{3}^{2}+\frac{1}{2} s_{4}^{2}+\frac{1}{2} y_{4}^{2}+\frac{1}{2} N_{3}^{2} \\
& +\left(\frac{1}{2}-k_{4}+\frac{3}{2} k_{5}\right) s_{4}^{2}+\frac{1}{2} N_{4}^{2}+\frac{1}{2} k_{5}-\tau_{3}^{-1} y_{3}^{2}+\frac{1}{2}\left(y_{3}^{2}+M_{3}^{2}\right)+-\tau_{4}^{-1} y_{4}^{2}+\frac{1}{2}\left(y_{4}^{2}+M_{4}^{2}\right)
\end{aligned}
$$

The design parameters should satisfy (Eq. 38):

$$
\left\{\begin{aligned}
k_{2} & \geq \frac{3}{2}+\frac{1}{2} \kappa, k_{3} \geq 2+\frac{1}{2} \kappa, k_{4} \geq \frac{3}{2} k_{5}+1+\frac{1}{2} \kappa \\
\tau_{3}^{-1} & \geq 1+\frac{1}{2} \kappa, \tau_{4}^{-1} \geq 1+\frac{1}{2} \kappa
\end{aligned}\right.
$$


where $\kappa$ is a constant, and $\kappa>0$. Thus, we have:

$$
\dot{V} \leq \kappa V+\hat{A}
$$

where $\hat{A}=\frac{1}{2} \tilde{N}_{2}^{2}+\frac{1}{2} N_{3}^{2}+\frac{1}{2} N_{4}^{2}+\frac{1}{2} M_{3}^{2}+\frac{1}{2} M_{4}^{2}+\frac{1}{2} k_{5}$.

From Eq. 39, we finally have (Eq. 40):

$$
V(t) \leq\left\{[\kappa V(0)-\widehat{A}] e^{-\kappa t}+\widehat{A}\right\} / \kappa
$$

Therefore, system convergence can be ensured by appropriately adjusting design parameters $k_{2}, k_{3}, k_{4}, k_{5}, \tau_{3}$, and $\tau_{4}$. If $k_{2}, k_{3}, k_{4}$, and $k_{5}$ are increased while $\tau_{3}$ and $\tau_{4}$ are reduced, a sufficiently large $\kappa$ can be ensured so that filtering error and error surface are sufficiently small. This ensures control accuracy.

\section{DESIGN OF DISTRIBUTED NETWORK BASED COOPERATIVE CONTROL STRATEGY}

The problem of the cooperative control of multiple intelligent agents can be described as a graph, which can be analyzed using graph theory. For the guidance and control of multiple interceptor missiles, one missile acquires the state information of other missiles through information exchange to achieve coordination among them. Therefore, a leader-follower topological structure that consists of one leading interceptor and $n$ following interceptors can be built. This structure is described using an undirected graph. Regarding each missile as a communication node, the information exchange between missiles is expressed as $\bar{G}=\{\bar{V}, \bar{E}, \bar{A}\}$, where $\bar{V}=\left\{\bar{v}_{1}, \bar{v}_{2}, \bar{v}_{3}, \ldots . ., \bar{v}_{n}\right\}$ represents the set formed by all missile nodes, $\bar{E}$ denotes the links between nodes, and $\bar{A}=\left[\bar{a}_{i j}\right] \in R^{n} \times^{n}$ is the adjacency matrix of the undirected graph. If information exchange exists between nodes $i$ and $j$, then $\bar{a}_{i j}>0$; otherwise, $\bar{a}_{i j}=0 . \bar{L}$ is the Laplace matrix of $\bar{G}$, the elements of which satisfy (Eq. 41 ):

$$
\left\{\begin{array}{l}
\bar{l}_{i i}=\sum_{j=1, j \neq i}^{n} \bar{a}_{i j} \\
\bar{l}_{i j}=-\bar{a}_{i j}, j \neq i
\end{array}\right.
$$

In a multi-missile topology with a leading interceptor, the leading interceptor features an independent state that does not change with followers. The purpose of including the leader state into the synchronization algorithm as part of the cooperative control strategy is that follower states should approach the leader state.

Let $\bar{B}=\operatorname{diag}\left\{\bar{b}_{1}, \bar{b}_{2}, \ldots . \bar{b}_{n}\right\}$ represent whether each follower can acquire the information of the leader, where $\bar{b}_{i}>0, i \in\{1,2,3, \ldots ., n\}$ implies yes and $\bar{b}_{i}=0, i \in\{1,2,3, \ldots ., n\}$ implies no.

Based on the fixed leader-follower topology, an improved cooperative control strategy for multiple interceptors can be designed using the distributed network method, as follows (Eq. 42):

$$
v_{i}=\bar{k}_{i 1}\left(\sum_{j=1}^{n} \bar{a}_{i j}\left(x_{j}-x_{i}\right)+\bar{b}_{i}\left(x_{0}-x_{i}\right)\right)+\bar{k}_{i 2}\left|\sum_{j=1}^{n} \bar{a}_{i j}\left(x_{j}-x_{i}\right)+\bar{b}_{i}\left(x_{0}-x_{i}\right)\right|^{\hat{D}_{i}} \operatorname{sgn}\left(\sum_{j=1}^{n} \bar{a}_{i j}\left(x_{j}-x_{i}\right)+\bar{b}_{i}\left(x_{0}-x_{i}\right)\right)+\dot{x}_{0}
$$

where $x_{0}$ denotes the position of the leader; $x_{i}, i \in\{1,2,3, \ldots ., n\}$ denotes the position of a follower; $v_{i}=\dot{x}_{i}$ denotes the velocity of a follower; $\partial_{i}>0 ; \bar{k}_{i 1}>0$ and $\bar{k}_{i 2}>0$ are constants.

Proof:

Lemma 1(Zou et al. 2010): Laplace matrix $\bar{L}$ has the following properties:

- If $\bar{G}$ is connected, the eigenvalue of $\bar{L}$ is, which is referred to as the algebraic connectivity of the graph; a larger value indicates a more connected network.

- $\quad 0$ is one eigenvalue of $\bar{L}$, and the corresponding eigenvector is 1 . 
Defining an error variable, $e_{i}=x_{i}-x_{0}$, we have (Eq. 43 ):

$$
\dot{e}_{i}=\dot{x}_{i}-\dot{x}_{0}=\bar{k}_{i 1}\left(\sum_{j=1}^{n} \bar{a}_{i j}\left(e_{j}-e_{i}\right)+\bar{b}_{i} e_{i}\right)+\bar{k}_{i 2}\left|\sum_{j=1}^{n} \bar{a}_{i j}\left(e_{j}-e_{i}\right)+\bar{b}_{i} e_{i}\right|^{\partial_{i}} \operatorname{sgn}\left(\sum_{j=1}^{n} \bar{a}_{i j}\left(x_{j}-x_{i}\right)+\bar{b}_{i}\left(x_{0}-x_{i}\right)\right)
$$

The Lyapunov function is defined as (Eq. 44):

$$
V=\frac{1}{2} e^{T}(\bar{L}+\bar{B}) e
$$

where $e=\left[e_{1}, e_{2}, \ldots, e_{n}\right]^{T}$.

We define $\bar{k}_{1}=\min \left\{\bar{k}_{i 1}\right\}, \bar{k}_{2}=\min \left\{\bar{k}_{i 2}\right\}$, and $\partial=\min \left\{\partial_{i}\right\}$. The derivatives of Eq. 44 are determined to obtain (Eq. 45):

$$
\begin{aligned}
\dot{V} & =\dot{e}^{T}(\bar{L}+\bar{B}) e=-\sum_{i=1}^{n}\left[\sum_{j=1}^{n} a_{i j}\left(e_{j}-e_{i}\right)-\bar{b}_{i} e_{i}\right] \dot{e}_{i}=-\sum_{i=1}^{n}\left[\sum_{j=1}^{n} a_{i j}\left(e_{j}-e_{i}\right)-\bar{b}_{i} e_{i}\right]\left[\bar{k}_{i 1}\left(\sum_{j=1}^{n} \bar{a}_{i j}\left(e_{j}-e_{i}\right)+\bar{b}_{i} e_{i}\right)+\right. \\
& +\bar{k}_{i 2}\left|\sum_{j=1}^{n} \bar{a}_{i j}\left(e_{j}-e_{i}\right)+\bar{b}_{i} e_{i}\right|^{\partial_{i}} \operatorname{sgn}\left(\sum_{j=1}^{n} \bar{a}_{i j}\left(x_{j}-x_{i}\right)+\bar{b}_{i}\left(x_{0}-x_{i}\right)\right]=-\sum_{i=1}^{n} \bar{k}_{i 1}\left[\sum_{j=1}^{n} a_{i j}\left(e_{j}-e_{i}\right)-\bar{b}_{i} e_{i}\right]^{2}-\sum_{i=1}^{n} \bar{k}_{i 2}\left[\sum_{j=1}^{n} a_{i j}\left(e_{j}-e_{i}\right)-\bar{b}_{i} e_{i}\right]^{1+\partial_{i}} \leq \\
& \leq-\bar{k}_{1} \sum_{i=1}^{n}\left[\sum_{j=1}^{n} a_{i j}\left(e_{j}-e_{i}\right)-\bar{b}_{i} e_{i}\right]^{2}-\bar{k}_{2} \sum_{i=1}^{n}\left[\sum_{j=1}^{n} a_{i j}\left(e_{j}-e_{i}\right)-\bar{b}_{i} e_{i}\right]^{1+\partial} \leq-\bar{k}_{1} \sum_{i=1}^{n}\left[\sum_{j=1}^{n} a_{i j}\left(e_{j}-e_{i}\right)-\bar{b}_{i} e_{i}\right]^{2}-\bar{k}_{2}\left(\sum_{i=1}^{n}\left[\sum_{j=1}^{n} a_{i j}\left(e_{j}-e_{i}\right)-\bar{b}_{i} e_{i}\right]^{2}\right)^{\frac{1+\partial}{2}}
\end{aligned}
$$

Considering $V(e) \neq 0$, Eq. 45 can be rewritten as:

$$
\frac{\sum_{i=1}^{n}\left[\sum_{j=1}^{n} a_{i j}\left(e_{j}-e_{i}\right)-\bar{b}_{i} e_{i}\right]^{2}}{V(e)}=\frac{e^{T}(\bar{L}+\bar{B})^{T}(\bar{L}+\bar{B}) e}{\frac{1}{2}\left(e^{T}(\bar{L}+\bar{B})^{T} e\right)} \geq 2 \lambda_{\min }(\bar{L}+\bar{B})
$$

From Eqs. 46 to 47:

$$
\dot{V}(t) \leq-\bar{k}_{1}\left[2 \lambda_{\min }(\bar{L}+\bar{B})\right]-\bar{k}_{2}\left[2 \lambda_{\min }(\bar{L}+\bar{B})\right]^{\frac{1+\partial}{2}}
$$

It is known from Lemma 1 that $V(t)$ can converge within finite time. That is, followers can converge to the state of the leader, achieving successful cooperative guidance and control for multiple interceptors.

\section{REALIZATION OF THE DISTRIBUTED NETWORK BASED COOPERATIVE CONTROL STRATEGY}

The essence of multi-interceptor cooperative attack is to coordinate the positions of following interceptors and the leading interceptor. Therefore, to realize the distributed network synchronization strategy, each interceptor in the network must follow the velocity commands provided by the synchronization strategy given by Eq. 42 .

The kinematic relations of the interceptors in the network that participate in coordinated attack are (Eq. 48):

$$
\left\{\begin{array}{l}
\dot{x}_{i}=V_{i} \cos \theta_{i} \\
\dot{y}_{i}=V_{i} \sin \theta_{i}
\end{array}\right.
$$

where $\dot{x}_{i}$ and $\dot{y}_{i}$ represent the two velocity components within the inertial frame of interceptor $i$ in the pitch channel.

Based on the distributed network synchronization strategy given in Eq. 42, the missile velocity reference commands are: 


$$
\left\{\begin{aligned}
\bar{V}_{m x i}= & \bar{k}_{i 1}\left(\sum_{j=1}^{n} \bar{a}_{i j}\left(x_{j}-x_{i}\right)+\bar{b}_{i}\left(x_{m}-x_{i}\right)\right) \\
& +\bar{k}_{i 2}\left|\sum_{j=1}^{n} \bar{a}_{i j}\left(x_{j}-x_{i}\right)+\bar{b}_{i}\left(x_{m}-x_{i}\right)\right|^{\partial_{i}} \\
& \operatorname{sgn}\left(\sum_{j=1}^{n} \bar{a}_{i j}\left(x_{j}-x_{i}\right)+\bar{b}_{i}\left(x_{m}-x_{i}\right)\right)+\dot{x}_{m} \\
\bar{V}_{m y i}= & \bar{k}_{i 1}\left(\sum_{j=1}^{n} \bar{a}_{i j}\left(y_{j}-y_{i}\right)+\bar{b}_{i}\left(y_{m}-y_{i}\right)\right) \\
& +\bar{k}_{i 2}\left|\sum_{j=1}^{n} \bar{a}_{i j}\left(y_{j}-y_{i}\right)+\bar{b}_{i}\left(y_{m}-y_{i}\right)\right|^{\partial_{i}} \\
& \operatorname{sgn}\left(\sum_{j=1}^{n} \bar{a}_{i j}\left(y_{j}-y_{i}\right)+\bar{b}_{i}\left(y_{m}-y_{i}\right)\right)+\dot{y}_{m}
\end{aligned}\right.
$$

Using Eqs. 48 and 49, the total velocity and flight path angle of the interceptor are obtained as (Eq. 50):

$$
\left\{\begin{array}{l}
\bar{V}_{m i}^{*}=\sqrt{\left(\bar{V}_{m x i}\right)^{2}+\left(\bar{V}_{m y i}\right)^{2}} \\
\bar{\theta}_{m i}^{*}=\arctan \left(\frac{\bar{V}_{m y i}}{\bar{V}_{m x i}}\right)
\end{array}\right.
$$

The signal must be filtered to obtain the derivative of the total velocity and flight path angle, $\dot{\bar{x}}$. Let $\bar{x}$ and $\bar{x}_{1}^{*}$ be the actual command and ideal command of the required signal, respectively. We obtain (Eq. 51):

$$
\ddot{\bar{x}}=-2 \zeta_{n} \omega_{n} \dot{\bar{x}}-\omega_{n}^{2} \bar{x}+\omega_{n}^{2} \bar{x}_{1}^{*}
$$

where $\zeta_{n}$ and $\omega_{n}$ are the damping and bandwidth of the filter, respectively. The use of the filter can effectively address the differentiation problem of the command signals without affecting the amplitudes of the commands and their derivatives.

\section{DESIGN OF FOLLOWING INTERCEPTOR CONTROLLER}

It can be seen that the commands provided by the cooperative control strategy can be converted to velocity and flight path angle commands. The controller for following interceptors adopts the dynamic surface SMC algorithm to achieve command signal tracking for the missiles in the cooperative network. Assuming that missile velocities are controllable and air resistance and gravity can be neglected, the flight velocity of a follower can be expressed as (Eq. 52):

$$
\dot{V}_{m}=\frac{\cos \alpha_{i}}{m} P_{i}
$$

where $P_{i}$ is engine thrust.

From Eq. 53, an error surface is defined as:

$$
s_{v}=V_{m}-\bar{V}_{m i}
$$

where $\bar{V}_{m i}$ is the filtered total velocity of the interceptor. The derivative of $s_{v}$ is obtained as (Eq. 54):

$$
\dot{s}_{v}=\frac{\cos \alpha_{i}}{m} P_{i}-\dot{\bar{V}}_{m i}
$$

where $\dot{\bar{V}}_{m i}$ is the derivative of $\bar{V}_{m i}$

The sliding mode reaching law provided below is used to ensure that interceptor velocity can rapidly follow the system command. 


$$
\dot{s}=-k_{a} s-k_{b}|s|^{\lambda} \operatorname{sgn}(s)
$$

Based on Eqs. 53 to 56, the engine thrust of a follower is (Eq. 56):

$$
P_{i}=\frac{m}{\cos \alpha_{i}}\left[\dot{\bar{V}}_{m i}-k_{v 1} s_{v}-k_{v 2}\left|s_{v}\right|^{\lambda_{v}} \operatorname{sgn}\left(s_{v}\right)\right]
$$

Let $x_{i 1}=\theta_{m i}, x_{i 2}=\alpha_{i}$, and $x_{i 3}=\omega_{i}$. The kinematic equation of interceptor $i$ in the pitch channel is (Eq. 57):

$$
\left\{\begin{array}{l}
\dot{x}_{i 1}=a_{i 23} x_{i 2}+d_{i 1} \\
\dot{x}_{i 2}=a_{i 33} x_{i 2}+d_{i 2} \\
\dot{x}_{i 3}=a_{i 43} x_{i 2}+a_{i 44} x_{i 3}+b_{i 3} u_{i}+d_{i 3}
\end{array}\right.
$$

where $\quad a_{i 23}=\frac{q_{i} S C_{y i}^{\alpha_{i}}}{m V_{m}}, \quad a_{i 33}=-\frac{q_{i} S C_{y i}^{\alpha_{i}}}{m V_{m}}, \quad a_{i 43}=\frac{q_{i} S \bar{L} m_{z i}^{\alpha_{i}}}{J_{z}}, \quad a_{i 44}=\frac{q_{i} S \bar{L} m_{z i}^{\omega_{z i}}}{J_{z}}, \quad b_{i 4}=\frac{1}{J_{z}}$.

Following the FTDO used above for the leader, an FTDO is designed to estimate disturbance $d_{i 1}$ (Eq. 58):

$$
\left\{\begin{array}{l}
\dot{z}_{i 10}=v_{i 10}+a_{i 23} x_{i 2}, \dot{z}_{i 11}=v_{i 11}, \dot{z}_{i 12}=v_{i 22} \\
v_{i 10}=-\lambda_{i 10} L_{i 1}^{1 / 3}\left|z_{i 10}-x_{i 1}\right|^{2 / 3} \operatorname{sgn}\left(z_{i 10}-x_{i 1}\right)+z_{i 11} \\
v_{i 11}=-\lambda_{i 11} L_{i 1}^{1 / 2}\left|z_{i 11}-v_{i 10}\right|^{1 / 2} \operatorname{sgn}\left(z_{i 11}-v_{i 10}\right)+z_{i 12} \\
v_{i 12}=-\lambda_{i 12} L_{i 1}\left|z_{i 12}-v_{i 11}\right|^{q_{i 1} / p_{i 1}} \operatorname{sgn}\left(z_{i 12}-v_{i 11}\right) \\
z_{i 10}=\tilde{x}_{i 1}, z_{i 11}=\tilde{d}_{i 1}, z_{i 12}=\dot{\tilde{d}}_{i 1}
\end{array}\right.
$$

where the estimated value of $d_{i 1}$ is $\tilde{d}_{i 1}$ and the estimation errors are $e_{i 11}=z_{i 11}-d_{i 1}$.

Similarly, unknown disturbances $d_{i 2}$ and $d_{i 3}$ are estimated with estimation errors of $e_{i 12}=z_{i 12}-d_{i 2}$ and $e_{i 13}=z_{i 13}-d_{i 3}$, respectively.

Based on the FTDO estimates and kinematic model in the pitch channel, the controller for following interceptors is designed using the dynamic surface SMC law.

- $\quad$ The first dynamic error surface is defined as (Eq. 59):

$$
s_{i 1}=a_{i 23}^{-1}\left(x_{i 1}-\bar{\theta}_{m}\right)
$$

The derivative of $s_{i 1}$ is obtained (Eq. 60):

$$
\dot{s}_{i 1}=x_{i 2}+a_{i 23}^{-1}\left(d_{i 1}-\dot{\bar{\theta}}_{m}\right)
$$

Based on the dynamic surface design method and FTDO, the virtual control input of the first dynamic surface is (Eq. 61):

$$
x_{i 2}^{*}=a_{i 23}^{-1}\left(-\tilde{d}_{i 1}+\dot{\bar{\theta}}_{m}\right)-k_{i 1} s_{i 1}
$$

where $k_{i 1}>0$. To prevent the increase in computational complexity owing to the 'explosion of terms' while finding the derivative of the virtual control input, $x_{3}^{*}$ is fed through a first-order low-pass filter to obtain the filtered virtual control input (Eq. 62):

$$
\tau_{i 2} \dot{\bar{x}}_{i 2}^{*}+\bar{x}_{i 2}^{*}=x_{i 2}^{*}, \bar{x}_{i 2}^{*}(0)=x_{i 2}^{*}(0) \quad \dot{\bar{x}}_{i 2}^{*}=-\tau_{i 2}^{-1}\left(\bar{x}_{i 2}^{*}-x_{i 2}^{*}\right)
$$


where $\tau_{i 2}>0$ is the time constant of the filter.

- The second dynamic error surface is defined as (Eq. 63):

$$
s_{i 2}=x_{i 2}-\bar{x}_{i 2}^{*}
$$

The derivate of $s_{i 2}$ is determined (Eq. 64):

$$
\dot{s}_{i 2}=a_{i 33} x_{i 2}+x_{i 3}+d_{i 2}-\dot{\bar{x}}_{i 2}^{*}
$$

Similar to the first dynamic surface, the virtual control input of the second dynamic surface is (Eq. 65):

$$
x_{i 3}^{*}=-a_{i 33} x_{i 2}-\tilde{d}_{i 2}+\dot{\bar{x}}_{i 2}^{*}-k_{i 2} s_{i 2}
$$

where $k_{i 2}>0 . x_{i 3}^{*}$ is fed through the following low-pass filter to obtain (Eq. 66):

$$
\begin{aligned}
& \tau_{i 3} \dot{\bar{x}}_{i 3}^{*}+\bar{x}_{i 3}^{*}=x_{i 3}^{*}, \bar{x}_{i 3}^{*}(0)=x_{i 3}^{*}(0) \\
& \dot{\bar{x}}_{i 3}^{*}=-\tau_{i 3}^{-1}\left(\bar{x}_{i 3}^{*}-x_{i 3}^{*}\right)
\end{aligned}
$$

where $\tau_{i 3}>0$ is the time constant of the filter.

- The third dynamic error surface is defined as (Eq. 67):

$$
s_{i 3}=x_{i 3}-\bar{x}_{i 3}^{*}
$$

The derivative of $s_{i 3}$ is determined (Eq. 68):

$$
\dot{s}_{i 3}=a_{i 43} x_{i 2}+a_{i 44} x_{i 3}+b_{i 3} u_{i}+d_{i 3}-\dot{\bar{x}}_{i 3}^{*}
$$

The following sliding mode reaching law is used to ensure a high rate of convergence for the system (Eq. 69):

$$
\dot{s}=-k_{a} s-k_{b}|s|^{\lambda} \operatorname{sgn}(s)
$$

The dynamic surface SMC law for following interceptors is (Eq. 70):

$$
u_{i}=b_{i 3}^{-1}\left[-a_{i 43} x_{i 2}-a_{i 44} x_{i 3}-\tilde{d}_{i 3}+\dot{\bar{x}}_{i 3}^{*}-k_{i 31} s_{i 3}-k_{i 32}\left|s_{i 3}\right|^{\lambda_{i 3}} \operatorname{sgn}\left(s_{i 3}\right)\right]
$$

where $k_{i 31}>0, k_{i 32}>0$, and $0<\lambda_{i 3}<1$.

The stability of the control algorithm for followers can be proved using Eqs. 20 to 40 .

\section{SIMULATION VALIDATION}

To validate the effectiveness of the improved distributed cooperative IGC algorithm, we assume a communication topology in which the leader can communicate with three other followers and neighboring communication exists between the followers, as shown in Fig. 2. The initial conditions of the leader, followers, and target are listed in Table 1.

The parameters of the FTDO are provided below.

1) FTDO parameters of the leader: $\lambda_{20}=\lambda_{30}=\lambda_{40}=2, \lambda_{21}=\lambda_{31}=\lambda_{41}=1.5, \lambda_{22}=\lambda_{32}=\lambda_{42}=2, q_{2}=q_{3}=q_{4}=0.5, p_{2}=p_{3}=p_{4}=8$, $\sigma_{20}=\sigma_{21}=\sigma_{22}=\sigma_{30}=\sigma_{31}=\sigma_{32}=\sigma_{40}=\sigma_{41}=\sigma_{42}=0.1, L_{2}=100, L_{3}=10$, and $L_{4}=50$. 


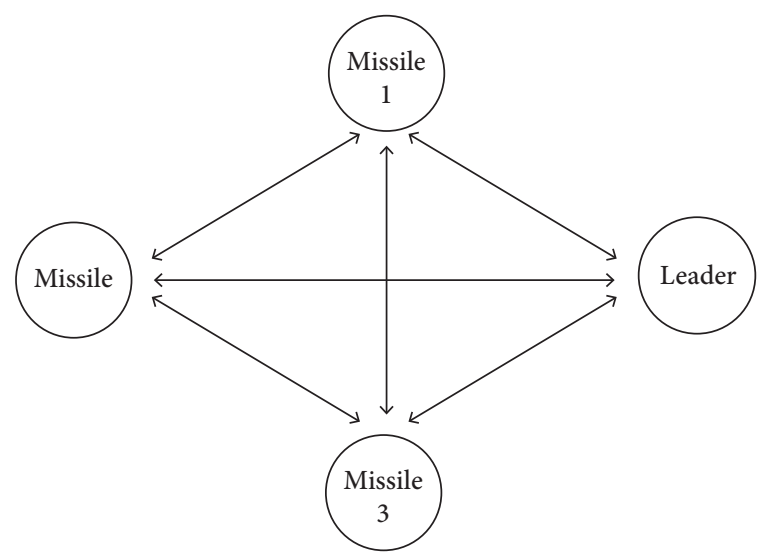

Figure 2. Communication topology of the leader and followers.

Table 1. Initial conditions of the leading interceptor, following interceptors, and target.

\begin{tabular}{|c|c|c|c|c|c|c|c|}
\hline No. & ID & Parameter & Value & Parameter & Value & Parameter & Value \\
\hline 1 & Leader & $x_{m 0}$ & $0 \mathrm{~m}$ & $y_{m 0}$ & $3300 \mathrm{~m}$ & $V_{m 0}$ & $400 \mathrm{~m} / \mathrm{s}$ \\
\hline 2 & Missile 1 & $x_{1 m 0}$ & $100 \mathrm{~m}$ & $y_{1 m 0}$ & $2400 \mathrm{~m}$ & $V_{1 m 0}$ & $400 \mathrm{~m} / \mathrm{s}$ \\
\hline 3 & Missile 2 & $x_{2 m 0}$ & $300 \mathrm{~m}$ & $y_{2 m 0}$ & $2700 \mathrm{~m}$ & $V_{2 m 0}$ & $400 \mathrm{~m} / \mathrm{s}$ \\
\hline 4 & Missile 3 & $x_{3 m 0}$ & $200 \mathrm{~m}$ & $y_{3 m 0}$ & $2600 \mathrm{~m}$ & $V_{3 m 0}$ & $400 \mathrm{~m} / \mathrm{s}$ \\
\hline 5 & Target & $x_{t 0}$ & $3000 \mathrm{~m}$ & $y_{t 0}$ & $380 \mathrm{~m}$ & $V_{t 0}$ & $240 \mathrm{~m} / \mathrm{s}$ \\
\hline
\end{tabular}

2) FTDO parameters of the followers: $\lambda_{i 10}=\lambda_{i 11}=\lambda_{i 12}=2, \lambda_{i 11}=\lambda_{i 21}=\lambda_{i 31}=1.5, \lambda_{i 12}=\lambda_{i 22}=\lambda_{i 32}=1.5, q_{i 1}=q_{i 2}=q_{i 3} 0.5$, $p_{i 1}=p_{i 2}=p_{i 3}=8$, and $L_{i 1}=L_{i 2}=L_{i 3}=10$.

Parameters of the dynamic surface SMC algorithm:

1) Parameters for the leader control law: $k_{2}=5, k_{3}=10, k_{4}=12, k_{5}=16, \partial=0.6$, and $x_{2 d}=0$.

2) Parameters for the follower control law: $k_{v 1}=k_{v 2}=2.5, k_{i 1}=5, k_{i 2}=2.5, k_{i 3}=10, k_{i 31}=40, k_{i 32}=5$, and $\lambda_{i 1}=\lambda_{i 3}=\lambda_{v}=0.6$.

Parameters for the filter: $\tau_{3}=\tau_{4}=\tau_{i 2}=\tau_{i 3}=0.002, \zeta_{n}=0.8$, and $\omega_{n}=40$.

Parameters for the distributed cooperative control strategy: $\bar{k}_{i 1}=0.2, \bar{k}_{i 2}=0.5$, and $\partial_{i}=0.6$.

It is assumed that the disturbance in the system is $d_{3}=d_{4}=d_{i 1}=d_{i 2}=d_{i 3}=0.1 \sin (t)$ and the target acceleration is $a=5 \mathrm{~m} / \mathrm{s}^{2}$.

The regular and improved distributed cooperative control strategies are examined in the simulations. The formula for the former is (Eq. 71):

$$
v_{i}=\bar{k}_{j 1}\left(\sum_{j=1}^{n} \bar{a}_{i j}\left(x_{j}-x_{i}\right)+\bar{b}_{i}\left(x_{0}-x_{i}\right)\right)+\dot{x}_{0}
$$

where $; \bar{k}_{j 1}>0.8$.

Simulation results are obtained using the parameters given above, and they are compared in Figs. 3 to 12 .

The trajectories of the interceptors and target obtained using the two strategies are shown in Figs. 3 and 8 . A comparison between the figures shows that when the improved strategy is used, follower trajectories gradually approach the target trajectory with a higher rate of convergence and smoother trajectory curves, and followers are able to hit the target by following the leader.

The velocity curves of the interceptors obtained using the two strategies are shown in Figs. 4 and 9. Convergence is reached at 6-8 s using the improved strategy, whereas it is reached at 8-10 s using the regular strategy. Comparisons between Figs. 5 and 10, 6 and 11, and 7 and 12 suggest that the improved strategy proposed in this study provides a higher rate of convergence and smoother transition with strong robustness. 


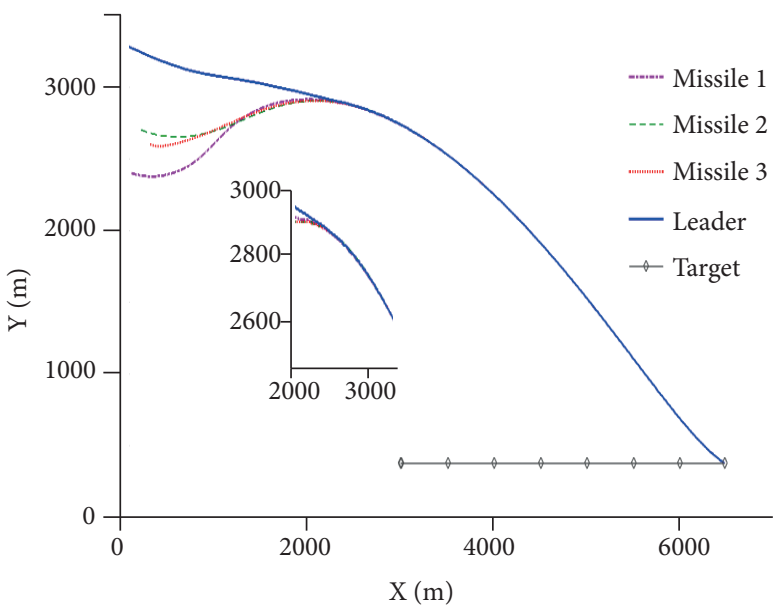

Figure 3. Trajectories of the interceptors and target obtained using the improved cooperative control strategy.

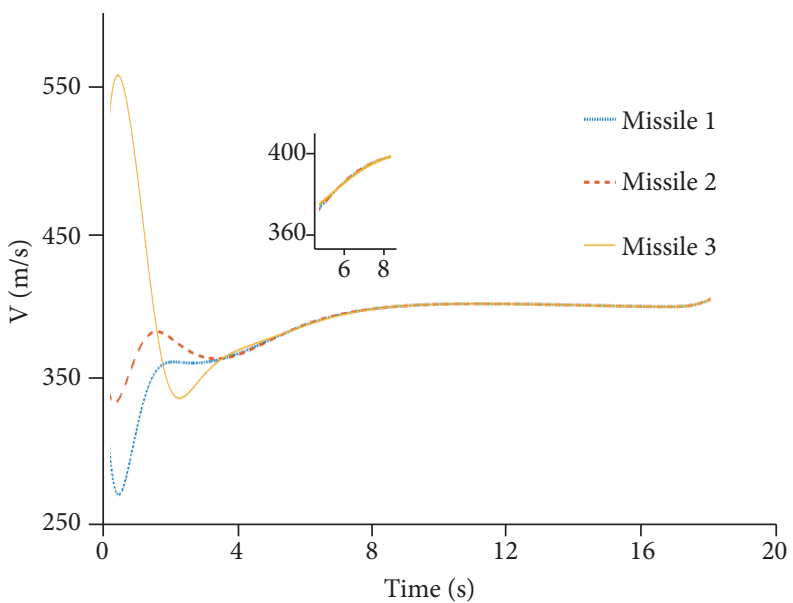

Figure 4. Velocity curves of the following interceptors obtained using the improved cooperative control strategy.

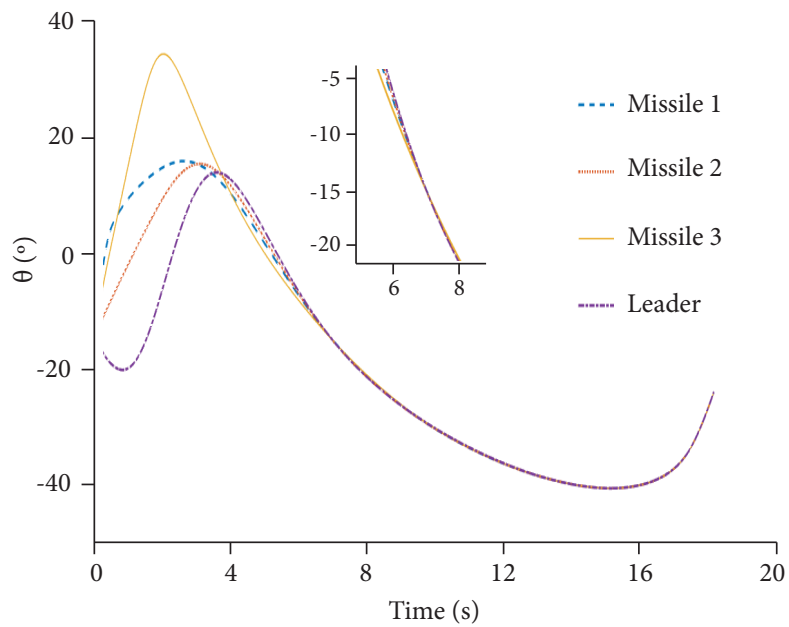

Figure 5. Flight path angles of the interceptors obtained using the improved cooperative control strategy. 


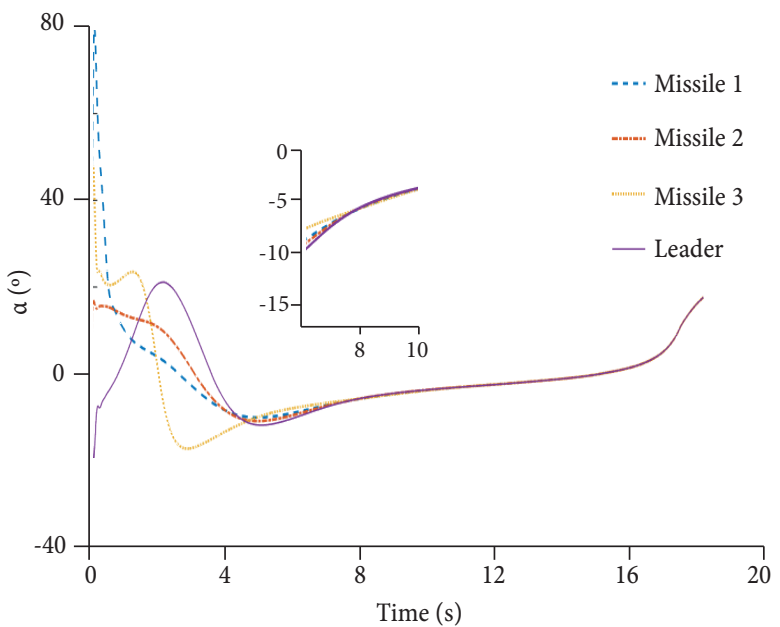

Figure 6. AOA curves of the interceptors obtained using the improved cooperative control strategy.

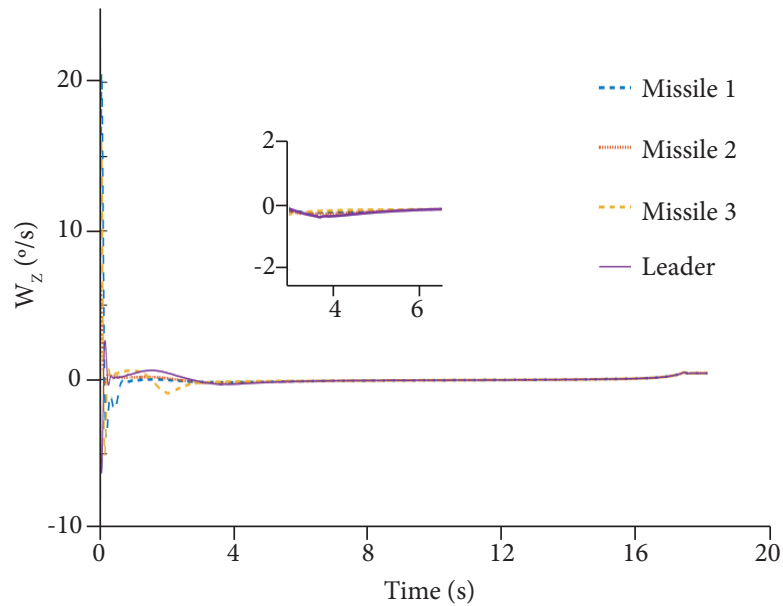

Figure 7. Pitch angular velocities of the interceptors obtained using the improved cooperative control strategy.

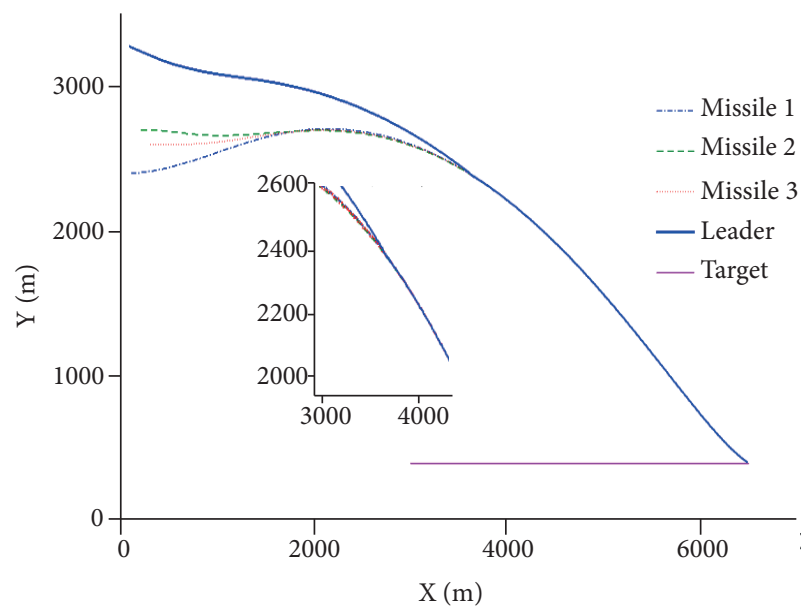

Figure 8. Trajectories of the interceptors and target obtained using the regular cooperative control strategy. 


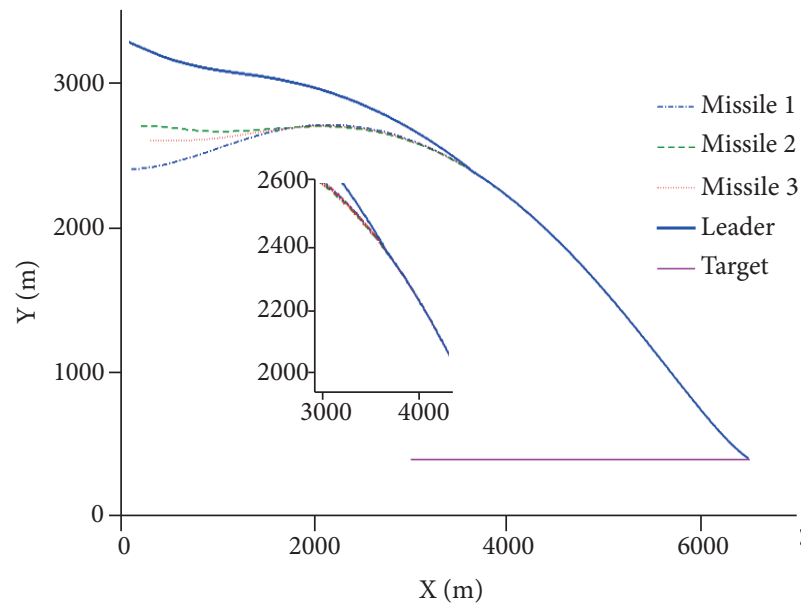

Figure 9. Velocity curves of the following interceptors obtained using the regular cooperative control strategy.

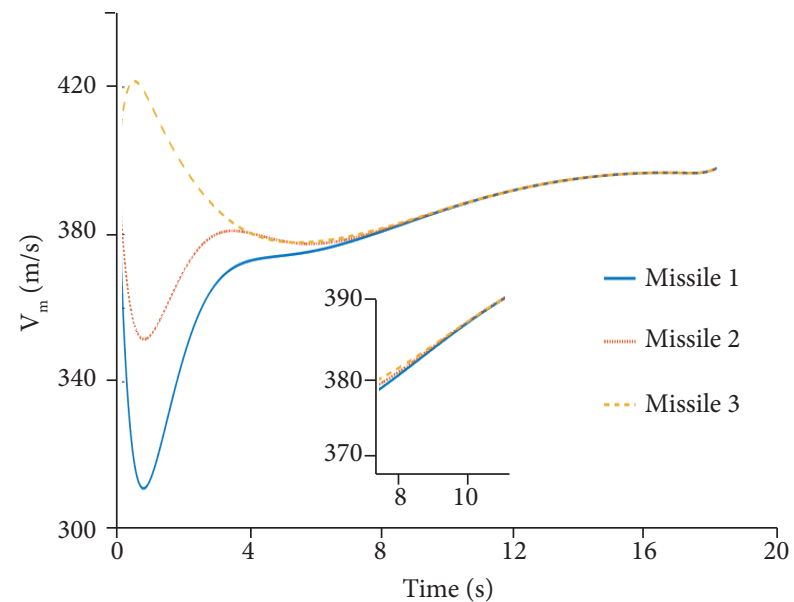

Figure 10. Flight path angles of the interceptors obtained using the regular cooperative control strategy.

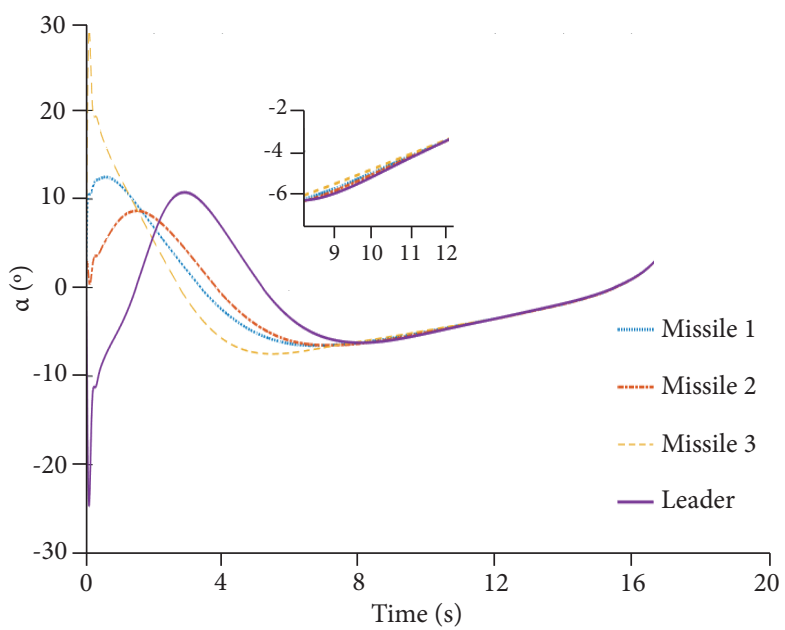

Figure 11. AOA curves of the interceptors obtained using the regular cooperative control strategy. 


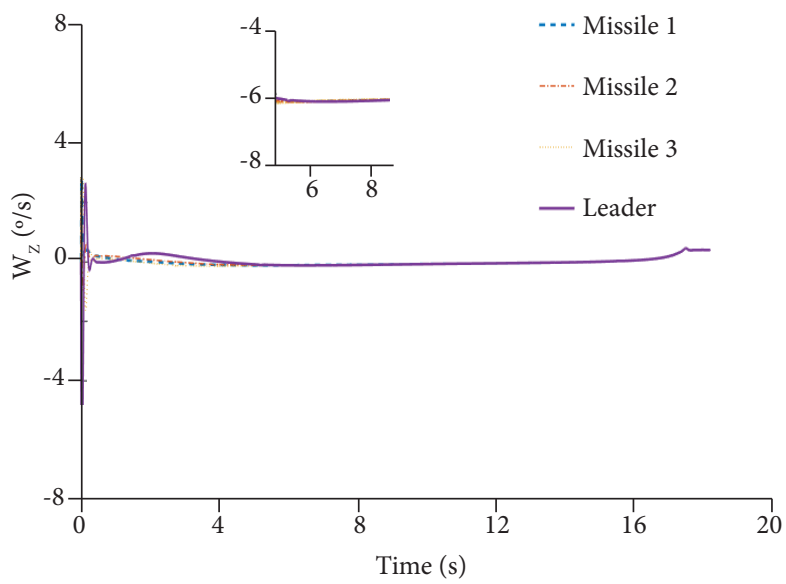

Figure 12. Pitch angular velocities of the interceptors obtained using the regular cooperative control strategy.

\section{CONCLUSION}

An improved distributed network cooperative IGC algorithm is developed based on the leader-follower topology to address the multi-interceptor IGC problem. The controller for the leading interceptor is designed based on an FTDO and the nonsingular fast dynamic surface SMC law, whose stability is proved using the Lyapunov principle. An improved multi-interceptor cooperative control strategy is proposed based on distributed network cooperative control. The following interceptor controller is similarly designed using an FTDO and dynamic surface SMC. The algorithm is validated using simulations. It is demonstrated that the developed algorithm can meet the cooperative guidance and control requirements of multiple interceptors while increasing the rate of convergence for the interceptors that react to cooperative control commands.

\section{AUTHORS' CONTRIBUTION}

Conceptualization, Liu X and Liang X; Methodology, Liu X; Investigation, Liu X; Writing - Original Draft, Liu X; Writing Review and Editing, Liu X and Liang X; Funding Acquisition, Liang X; Resources, Liang X; Supervision, Liang X.

\section{FUNDING}

Aeronautical Science Foundation of China [http://dx.doi.org/10.13039/501100004750]

Grant No 2016ZC12005

\section{REFERENCES}

Balhance N, Weiss M, Shima T (2017) Cooperative guidance law for intrasalvo tracking. Journal of Guidance, Control, and Dynamics 40(6):1441-1456. https://doi.org/10.2514/1.6002250

Cho D, Kim HJ, Tahk MJ (2016) Nonsingular sliding mode guidance for impact time control. Journal of Guidance, Control, and Dynamics 39(1):61-68. https://doi.org/10.2514/1.G001167 
Daughtery E, Qu ZH (2014) Optimal design of cooperative guidance law for simultaneous strike. Presented at: 53rd IEEE Conference on Decision and Control; Los Angeles, USA. https://doi.org/10.1109/CDC.2014.7039510

Harl N, Balakrishnan SN (2012) Impact time and angle guidance with sliding mode control. J IEEE Transactions on Control Systems Technology 20(6):1436-1449. https://doi.org/10.1109/TCST.2011.2169795

Jeon IS, Lee Jl, Tahk MJ (2010) Homing guidance law for cooperative attack of multiple missiles. J Journal of Guidance, Control, and Dynamics 33(1):275-280. https://doi.org/10.2514/1.40136

Kang S, Kim HJ (2011) Differential game missile guidance with impact angle and time constrain. IFAC Proceedings Volumes 44(1):39203925. https://doi.org/10.3182/20110828-6-IT-1002.00805

Kumar SR, Ghose D (2014) Cooperative rendezvous guidance using sliding mode control for interception of stationary targets. IFAC Proceedings Volumes 47(1):447-483. https://doi.org/10.3182/20140313-3-IN-3024.00154

Lee Jl, Jeon IS, Tahk MJ (2007) Guidance law to control impact time and angle. IEEE Transactions on Aerospace and Electronic Systems 43(1):301-310. https://doi.org/10.1109/TAES.2007.357135

Lee Y, Kim Y, Moon G, Jun BE (2016) Sliding mode based missile integrated attitude control schemes considering velocity change. Journal of Guidance, Control, and Dynamics 39(3):423-436. https://doi.org/10.2514/1.G001416

Menon PK, Ohlmeyer EJ (2001) Integrated Design of Agile Missile Guidance and Autopilot Systems. Control Engineering Practice 9(10):1095-1106. https://doi.org/10.1016/S0967-0661(01)00082-X

Nikusokhan M, Nobahari H (2016) Closed-form optimal cooperative guidance law against random step maneuver. IEEE Transactions on Aerospace and Electronic Systems 52(1):319-336. https://doi.org/10.1109/TAES.2015.140623

Ran MP, Wang Q, Hou DL, Dong C (2014) Backstepping Design of Missile Guidance and Control Based on Adaptive Fuzzy Sliding Mode Control. Chinese Journal of Aeronautics 27(3):634-642. https://doi.org/10.1016/i.cja.2014.04.007

Seyedipour SH, Jegarkandi MF, Shamaghdari S (2017) Nonlinear integrated guidance and control based on adaptive back-stepping scheme. Aircraft Engineering and Aerospace Technology 89(3):415-424. https://doi.org/10.1108/AEAT-12-2014-0209

Shaferman V, Shima T (2015) Cooperative optimal guidance laws for imposing a relative intercept angle. Journal of Guidance, Control, and Dynamics 38(8): 1395-1408. https://doi.org/10.2514/1.G000568

Shamaghdari S, Nikravesh SKY, Haeri M (2015) Integrated guidance and control of elastic flight vehicle based on robust MPC. International Journal Of Robust And Nonlinear Control 25(15):2608-2630. https://doi.org/10.1002/rnc.3215

Shtessel YB, Shkolnikov IA, Levant A (2007) Smooth second-order sliding modes: missile guidance application. Automatica 43(8):14701476. https://doi.org/10.1016/j.automatica.2007.01.008

Shtessel YB, Shkolnikov IA, Levant A (2009) Guidance and control of missile interceptor using second-order sliding modes. IEEE Transactions on Aerospace and Electronic Systems 45(1):110-124. https://doi.org/10.1109/TAES.2009.4805267

Sun XJ, Zhou R, Hou DL, Wu J (2014) Consensus of leader-followers system of multi-missile with time-delays and switching topologies. Optik 125(3):1202-1208. https://doi.org/10.1016/j.ijleo.2013.07.159

Vaddi SS, Menon PK, Ohlmeyer EJ (2009) Numerical State-dependent Riccati Equation Approach for Missile Integrated Guidance Control. Journal of Guidance, Control and Dynamics 32(2):699-703. https://doi.org/10.2514/1.34291

Wang XF, Zheng YY, Lin H (2015) Integrated guidance and control law for cooperative attack of multiple missile. Aerospace Science and Technology 42:1-11. https://doi.org/10.1016/i.ast.2014.11.018

Zhao E, Wang S, Chao T, Yang M (2014) Multiple missiles cooperative guidance based on leader-follower strategy. Presented at: 2014 IEEE Chinese Guidance, Navigation and Control Conference; Yantai, China. https://doi.org/10.1109/CGNCC.2014.7007366

Zhao JB, Yang SX (2017) Review of muti-missile cooperative guidance. Acta Aeronautica et Astronautica Sinica 38(1):20256. https:// doi.org/10.7527/S1000-6893.2016.0136

Zhao QL, Chen J, Dong XW, Li Q, Ren Z (2016) Cooperative guidance law for heterogeneous missiles intercepting hypersonic weapon. Acta Aeronautica et Astronautica Sinica 37(3):936-948. https://doi.org/10.7527/S1000-6893.2015.0235

Zou L, Ding QX, Zhou R (2010) Distribute cooperative guidance for multiple heterogeneous networked missiles. Journal of Beijing University of Aeronautics and Astronautics 36(12):1432-1435. https://doi.org/10.13700/j.bh.1001-5965.2010.12.023 